\title{
X-Ray Lasers Film Chemical Reaction in Super-Slow Motion
}

\author{
Extremely brilliant, extremely short x-ray pulses let researchers film a \\ femtosecond-resolution movie of a chemical reaction, revealing \\ unexpected dynamics.
}

\section{By Sophia Chen}

$\Gamma$

he ability to generate extremely bright, ultrashort x-ray pulses in recent years has allowed researchers to study the fleeting dynamics of chemical reactions, which occur on femtosecond $\left(10^{-15} \mathrm{~s}\right)$ timescales. Now, Sebastian Westenhoff of the University of Gothenburg, Sweden, and colleagues have combined femtosecond time resolution with sub-angstrom $\left(10^{-10} \mathrm{~m}\right)$ spatial resolution in a single experiment [1]. In so doing, they have obtained new insight into how a certain well-studied molecular photodissociation reaction unfolds.

The team used two different x-ray free-electron lasers-the Linac Coherent Light Source in the US and the SPring-8 Angstrom Compact Free Electron Laser in Japan-to "film" a molecule of diiodemethane breaking apart and recombining in the solvent cyclohexane. The resulting movie shows how, over the course of a nanosecond, the molecule is triggered by an ultraviolet light pulse to split into two radicals. Combining their

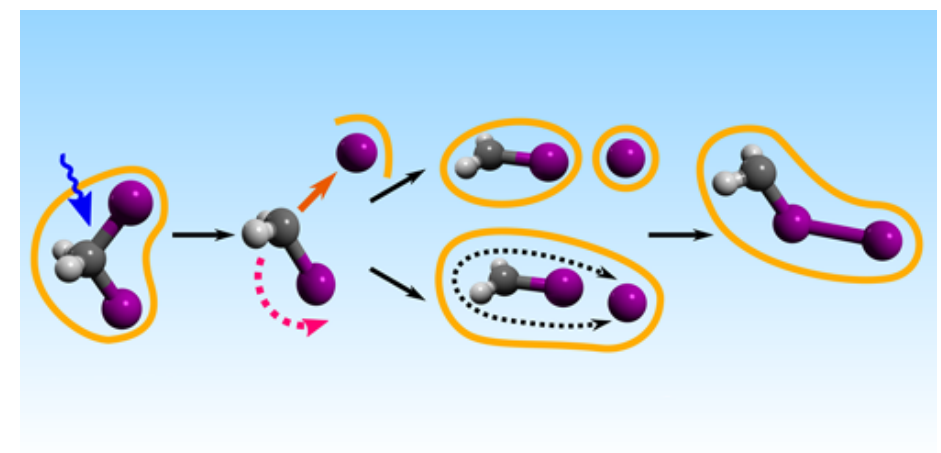

Credit: M. Panman/University of Gothenburg recording with simulations, the researchers were able to track the positions of the two radicals with new precision as they collided with the solvent before reacting to form a structurally different photoisomer.

From their analysis, the researchers determined how the radicals' translational and rotational kinetic energies are dispersed into the solvent-a process not detectable by earlier optical spectroscopic measurements. They found that the two radicals' translational motion decreases quickly. This leads to the radicals being confined in the same solvent "cage," where they remain for an unexpectedly long time.

The detailed dynamics of the radicals' motion in the period after photodissociation influences their downstream recombination reactions. Movies that capture these dynamics therefore provide a valuable new technique for studying such reactions and could eventually help scientists engineer chemical processes on precise time and spatial scales.

Sophia Chen is a freelance science writer based in Columbus, Ohio.

\section{REFERENCES}

1. M. Panman et al., "Observing the structural evolution in the photodissociation of diiodomethane with femtosecond solution x-ray scattering," Phys. Rev. Lett. 125, 226001 (2020). 